\title{
Evaluation of the combined antimicrobial activity of the leaf extract of phyllantus muellerianus with ciprofloxacin
}

\author{
Kenneth C. Ofokansi', Anthony A. Attama', Philip F. Uzor ${ }^{2 *}$ and Mercy O. Ovri ${ }^{1}$ \\ *Correspondence: philuzor4u@yahoo.com \\ 'Department of Pharmaceutics, Faculty of Pharmaceutical Sciences, University of Nigeria, 410001, Nsukka, Enugu State, Nigeria. \\ ${ }^{2}$ Department of Pharmaceutical and Medicinal Chemistry, University of Nigeria, 410001, Nsukka, Enugu State, Nigeria.
}

\begin{abstract}
Background: Infectious diseases caused by multi-resistant microbial strains are on the increase. Fighting these diseases with natural products may be more efficacious. The aim of this study was to investigate the in vitro antimicrobial activity of methanol leaf extract and chromatographic fractions of the extract of Phyllanthus muellerianus (Kuntze) Excell against Klebsiella spp., Enterococcus faecalis, Pseudomonas earuginosa, Proteus mirabilis, Escherichia coli, Salmonella spp. and Candida albicans. The combined effect of the extract or its fractions with ciprofloxacin was also tested.

Methods: The antimicrobial properties of the methanol extract of $P$. muellerianus and its fractions were determined in vitro using the agar diffusion method. Agar diffusion Checkerboard method and Overlay Inoculum susceptibility disc method were used to study the interaction of the extract or fraction with ciprofloxacin. The extract was subjected to phytochemical analysis for identification of constituents using standard procedures. The minimum inhibitory concentration (MIC) data obtained from the antimicrobial studies were analyzed for significant difference at $\mathrm{p}<0.05$ using one way analysis of variance (ANOVA).

Results: While n-hexane and methylene chloride fractions showed no activity against the test microorganisms, methanol crude extract and methanol fraction of the extract revealed a good concentration dependent antibacterial activity against $P$. earuginosa, $P$. mirabilis and $E$. faecalis. Combinations of the extract and ciprofloxacin produced antagonistic effect against $P$. mirabilis and $E$. faecalis while a synergistic effect was obtained against $P$. aeruginosa. The results of the phytochemical screening showed that the extract and the fractions contain glycosides, flavoniods, saponins, alkaloids. Conclusion: The methanol leaf extract of Phyllanthus muellerianus possess antibacterial activity against $P$. earuginosa, $P$. mirabilis and E. faecalis and could possibly be used in combination with ciprofloxacin to treat infections caused by P. aeruginosa.
\end{abstract}

Keywords: Antimicrobial activity, ciprofloxacin, Enterococcus faecalis, Phyllanthus muellerianus, Proteus mirabilis, Pseudomonas earuginosa

\section{Background}

Infectious disease account for approximately one half of all deaths in tropical countries [1] and they are considered a major threat to human health because of the unavailability of vaccines or limited chemotherapy. Ranked $5^{\text {th }}$ in 1981 , infectious diseases continue to be a growing public health concern [2] and they became the $3^{\text {rd }}$ leading cause of death in 1992, with an increase of $58 \%$ [3]. Most of the current antibiotics have considerable limitations in terms of antimicrobial spectrum, side effects and their widespread overuse has led to increasing clinical resistance of previously sensitive microorganisms and to the occurrence of uncommon infections [4]. The use of medicinal plants as a source of relief from illness can be traced back over 5 millennia to written documents of the early civilization in China, India and the near East, but it is doubtless an art as old as mankind. Neanderthals living 6,000 years ago in present day Iraq used plants such as hollyback; which are still widely used in ethno medicine around the world [5]. With an advent of ever-increasing resistant bacteria and fungi strains, there has been a corresponding rise in the universal demand for natural antimicrobial therapeutics. Herbal medicines have widely been used and now form an integral part of the primary health care in many countries [6]. Plants used for traditional medicines contain a wide range of substances that can be used to treat chronic as well as acute infectious diseases $[7,8]$, and have therefore become sources of important drugs and the pharmaceutical industries have come to consider traditional medicine as a source of bioactive agents that can be used in the preparation of synthetic medicines.

Phyllanthus muellerianus (Kuntz) Excel; is a glabrous shrub or woody climber occasionally arborescent, found in Savannah and drier secondary forests. Common in coastal thicket and widespread in parts of Tropical Africa, Phyllanthus muellerianus is widely used to treat intestinal troubles, severe dysentery, constipation, stomach ache, jaundice, urethral discharges and as wound dressing [9]. The leaf extract is used as a vapour bath to treat veneral diseases and toothache [10].

The antibacterial activities of the plant have been

(C) 2013 Uzor et al; licensee Herbert Publications Ltd. This is an Open Access article distributed under the terms of Creative Commons Attribution License (http://creativecommons.org/licenses/by/3.0). This permits unrestricted use, distribution, and reproduction in any medium, provided the original work is properly cited. 
demonstrated in the leaf and stem back of the plant against some selected bacteria $[11,12]$. However, in the present research the antimicrobial properties of methanolic extract of Phyllanthus muellerianus and their various chromatographic fractions ( $n$-hexane, methylene chloride and methanol) were evaluated and studied singly and in combination with ciprofloxacin, a broad spectrum antibiotic, against a wider range of microorganisms such as Klebsiella spp., Enterococcus faecalis, Pseudomonas earuginosa, Proteus mirabilis, Escherichia coli, Salmonella spp and Candida albicans. The above organisms were chosen because they are ubiquitous in our environment and are the causative agents of most common infections. The standard antibiotic ciprofloxacin, was also chosen due to the fact that it is one the antibiotics usually prescribed for most common infections and it is quite common to see a patient taking herbal medications and standard antibiotics concurrently.

\section{Methods \\ Plant material}

The leaves of the plant P. muellerianus were collected from Nsukka in Enugu State between the months of June and July, 2010 and were authenticated by Mr. A. O. Ozioko of Bioresource Development and Conservative Program Centre (BDCP) in Nsukka Enugu State, Nigeria. The voucher specimen for identification was deposited at the center.

\section{Extraction of plant material}

The pulverized dry leaves were extracted with a Soxhlet extractor using methanol (Sigma-Aldrich, Germany) as the solvent. The extract recovered was evaporated to dryness under room temperature. The methanolic crude extract $(40$ g) was dissolved in about $400 \mathrm{ml}$ of methanol and adsorbed onto silica gel (50 - 200, Lab Tech Chemicals) and eluted in succession with $\mathrm{n}$-hexane, methylene chloride and methanol in order of increasing polarity. The solvent was removed by evaporation to obtain solvent free fractions.

\section{Phytochemical tests}

Phytochemical analysis of the crude methanol extract (ME) together with $n$-hexane (NF), methylene chloride (MCF) and methanol (MF) fractions of the extract was carried out based on a procedure described by Sofowora [13] and Trease and Evans [14].

\section{Microbiological evaluation}

Five isolates each of the following organisms were used: Klebsiella spp., Enterococcus faecalis, Pseudomonas earuginosa Proteus mirabilis, Escherichia coli, Salmonella spp. and Candida albicans. They were isolated from patients who visited Adonai Diagnostic and Research Laboratory in Nsukka, Enugu State. Isolation and characterization was done according to the method described $[15,16]$. The stock culture of each clinical isolates of bacteria was stored in nutrient agar slants at $4^{\circ} \mathrm{C}$. Prior to use, the cultures were activated by successive daily sub-culturing first to MacConkey agar and then into nutrient agar slant to ensure there was no contamination. Candida albicans isolates were inoculated onto Sabouraund's dextrose agar plates. The standardization of the various bacteria inoculums was carried out according to the method described by Arora [17].

\section{Antimicrobial screening test}

Antimicrobial screening of the methanol crude extract (ME) and fractions of the Phyllanthus muellerianus and standard antibiotic (ciprofloxacin) against the bacteria was done by cup-plate agar diffusion method $[18,19]$. A known concentration $(200 \mathrm{mg} / \mathrm{ml})$ of each of the extract and fractions in DMSO was serially diluted twofold. Molten nutrient agar already prepared according to the manufacturer's protocol was seeded with $0.1 \mathrm{ml}$ of standardized broth cultures of the microorganisms. For each microbial isolate, wells, $8 \mathrm{~mm}$ in diameter, were made in the solid agar using a sterile cork borer. Solutions of ciprofloxacin (for bacteria) and nystatin (for fungi) were added into separate agar-wells as positive control while the centre wells contained DMSO as negative control. The other wells were filled with various concentrations of the respective test agents. All the plates were left for $1 \mathrm{~h}$ at room temperature for proper diffusion after which they were incubated at $37^{\circ} \mathrm{C}$ for $24 \mathrm{~h}$ (for bacteria) and $48 \mathrm{~h}$ (for fungi). The inhibition zone diameter (IZD) was measured in each case.

\section{Evaluation of the minimum inhibitory concentration} (MIC)

The MIC of the antimicrobial agents was determined using agar diffusion method [20] by plotting the graph of the $I_{Z D}\left(\mathrm{~mm}^{2}\right)$ against the log of concentration and the point of intersection on the $\mathrm{x}$-axis was recorded as the MIC.

\section{In vitro evaluation of the extracts and the standard antibiotic in combination}

The effects of the extracts in combination with ciprofloxacin against the bacteria were evaluated using agar diffusion Checkerboard method and the Overlay Inoculum susceptibility disc method as described by Okore [21]. In the agar diffusion, concentrations of the extract (ME) or fraction (MF) and ciprofloxacin were prepared according to a continuous variation Checkerboard technique starting from $0: 10$ (that is, 0 part of ciprofloxacin to 10 part of ME), then 1:9, 2:8 ...10:0. The MIC of each combination was determined as described above. For each isolate, the fractional inhibitory concentrations (FIC) of all ratios of the combined extract and ciprofloxacin was determined and the FIC value for each extract was calculated using Equation 1:

$$
\text { FIC (extract } A)=\frac{\text { MIC of the extract } A \text { in combination }}{\text { MIC of } A \text { alone }}
$$


Ofokansi et al. Journal of Pharmaceutical Technology \& Drug Research 2013,

http://www.hoajonline.com/journals/pdf/2050-120X-2-16.pdf

The sum of FIC A and FIC B gives the FIC Index (Equation 2) from where an inference can be drawn:

$\mathrm{FIC}$ Index = FIC A + FIC B Equation 2.

The effects of the combinations were classified as synergistic, additive, indifference and antagonistic, if the FIC Index $<1$, $=1,>1 \leq 2$ and $>2$ respectively [21]. For the Overlay Inoculum agar susceptibility technique, a sub-inhibitory concentration of one of the test agents for the combination was prepared in molten nutrient agar. The antibiotic agar was poured in sterile clean Petri dish and allowed to solidify to form the base antibiotic agar. About $2 \mathrm{ml}$ of molten nutrient agar was inoculated with the test organism and shaken gently to ensure uniformity of the cells in the medium. The inoculated medium was then poured on the surface of the base antibiotic agar to form a thin uniform layer, the overlay inoculum agar. When the inoculum agar has solidified, the second antibiotic in the form of an antibiotic disc was placed on the agar medium (ciprofloxacin). Two control plates were prepared. Control A contained only the base antibiotic and the overlay inoculum agar, while control B contained the base agar without antibiotic, the overlay inoculum and the antibiotic disc. The three plates in the set were kept at room temperature for $1 \mathrm{~h}$ to allow for pre-diffusion, and then incubated at $37^{\circ} \mathrm{C}$ for $24 \mathrm{~h}$. The zone of inhibition formed in the test plates was used to determine the combined effect of the two antibiotics, by comparing it with the result in control B. Synergism is obtained when the diameter of the zone of inhibition in the test plate is greater than that in control B by at least $19 \%$, lower than $19 \%$ indicates additivity, equal to control $B$ indicates indifference; when it is less than control $B$, there is antagonism.

\section{Statistical analysis}

All values were expressed as mean \pm SEM and difference between the means of MIC and FIC (Index) were considered significant at $p<0.05$ using one way analysis of variance (ANOVA). SPSS package version 18.0 for Windows was used for the analysis.

\section{Results}

\section{Phytochemical analysis}

The results of the phytochemical composition of the extract and fractions of the leaves of $P$. muellerianus show that the most common substance found in the extract and fractions were glycosides, alkaloids, steroids, terpenoids and flavonoids while proteins were absent in the extract and the fractions.

\section{Antimicrobial screening test}

The results of the sensitivity test of the extract and fractions and the standard drug (ciprofloxacin) against the microorganisms are presented in Table 1. Methylene chloride fraction (MCF) and n-hexane fraction (NF) did not show any activity against all the bacterial isolates used
Table 1. IZD produced by the extract and ciprofloxacin against sensitive microorganisms.

\begin{tabular}{lcccc}
\hline Treatment & Dose $(\mathrm{mg} / \mathrm{ml})$ & \multicolumn{3}{c}{ IZD $(\mathrm{mm})^{\mathrm{a}}$} \\
\hline MF & & P. aeruginosa & P. mirabilis & E. faecalis \\
\hline & 200.0 & $11.20 \pm 1.01$ & $9.75 \pm 0.22$ & $12.40 \pm 1.02$ \\
& 100.0 & $8.80 \pm 0.86$ & $8.50 \pm 0.43$ & $10.00 \pm 0.44$ \\
& 50.0 & $5.80 \pm 1.62$ & $6.50 \pm 0.25$ & $7.80 \pm 0.37$ \\
& 25.0 & $1.80 \pm 0.80$ & 0 & $2.80 \pm 1.74$ \\
ME & 12.5 & $1.20 \pm 0.20$ & 0 & 0 \\
& 200.0 & $11.80 \pm 0.37$ & $13.75 \pm 0.22$ & $16.60 \pm 1.02$ \\
& 100.0 & $9.40 \pm 0.40$ & $10.25 \pm 0.41$ & $13.60 \pm 1.36$ \\
& 50.0 & $7.60 \pm 0.24$ & $7.75 \pm 0.22$ & $10.80 \pm 0.96$ \\
& 25.0 & $6.40 \pm 0.24$ & $7.00 \pm 0.61$ & $8.20 \pm 1.06$ \\
& 12.5 & $2.40 \pm 1.46$ & 0 & $4.60 \pm 1.98$ \\
& 2.000 & $30.80 \pm 1.35$ & $30.00 \pm 1.31$ & $18.00 \pm 1.51$ \\
& 1.000 & $27.20 \pm 1.31$ & $26.80 \pm 1.32$ & $13.20 \pm 1.06$ \\
& 0.500 & $25.00 \pm 1.40$ & $24.00 \pm 1.19$ & $10.00 \pm 1.67$ \\
0.250 & $21.60 \pm 0.40$ & $20.00 \pm 1.75$ & $3.80 \pm 2.33$ \\
0.125 & $17.60 \pm 1.50$ & $15.20 \pm 1.73$ & 0 \\
\hline
\end{tabular}

${ }^{a}$ Mean \pm SD ( $\left.=5\right)$; MF - Methanol fraction;

ME - Methanol crude extract; Cipro - Ciprofloxacin.

Table 2. MIC values of the extracts and ciprofloxacin against sensitive microorganisms.

\begin{tabular}{lccc}
\hline Microorganism & \multicolumn{3}{c}{$\mathrm{MIC}(\mathbf{m g} / \mathbf{m l})^{\mathrm{b}}$} \\
\hline P. aeruginosa & $21.05 \pm 4.93$ & $11.41 \pm 1.99$ & $0.05 \pm 0.01$ \\
P. mirabilis & $12.41 \pm 3.49$ & $17.53 \pm 1.43$ & $0.03 \pm 0.01$ \\
E. faecalis & $15.76 \pm 1.49$ & $11.82 \pm 2.66$ & $0.33 \pm 0.05$ \\
\hline
\end{tabular}

${ }^{b}$ Values are Mean $\pm \mathrm{SD}(\mathrm{n}=5)$;

${ }^{\mathrm{c}} \mathrm{MIC}$ values are significantly $(\mathrm{p}<0.05)$ different from the MIC of control (ciprofloxacin).

and their results are not presented in the table. Methanol fraction (MF) and the methanol crude extract (ME) did not show any activity against the isolates of Klebsiella spp., E.coli and Salmonella spp. but produced clear zones on all the isolates of $P$. aeruginosa, $P$. mirabilis and $E$. faecalis. None of the extracts showed activity against Candida albicans. The IZDs produced by MF and ME against different isolates of $P$. aeruginosa were concentration dependent, ranging from 1.20 (at $12.25 \mathrm{mg} / \mathrm{ml}$ ) to $11.20 \mathrm{~mm}$ (at $200 \mathrm{mg} / \mathrm{ml}$ ) and 2.40 (at $12.25 \mathrm{mg} / \mathrm{ml}$ ) to $11.80 \mathrm{~mm}$ (at $200 \mathrm{mg} / \mathrm{ml}$ ) respectively. Similarly, the IZD produced by MF and ME against $P$. mirabilis ranged from zero inhibition (at 12.25 and $25.50 \mathrm{mg} / \mathrm{ml}$ ) to $9.75 \mathrm{~mm}$ (at $200 \mathrm{mg} / \mathrm{ml}$ ) and zero inhibition (at 12.25 $\mathrm{mg} / \mathrm{ml}$ ) to $13.75 \mathrm{~mm}$ (at $200 \mathrm{mg} / \mathrm{ml}$ ) respectively. The IZD for the $E$. faecalis varied ranging from zero inhibition (at $12.25 \mathrm{mg} / \mathrm{ml}$ ) to $12.40 \mathrm{~mm}$ (at $200 \mathrm{mg} / \mathrm{ml}$ ) and $4.60 \mathrm{~mm}$ (at $12.5 \mathrm{mg} / \mathrm{ml}$ ) to $16.60 \mathrm{~mm}$ (at $200 \mathrm{mg} / \mathrm{ml}$ ) for MF and ME respectively. The IZD for the control, ciprofloxacin, ranged from zero inhibition (at $0.125 \mathrm{mg} / \mathrm{ml}$ for E. faecalis) to $30.80 \mathrm{~mm}$ (at $2.0 \mathrm{mg} / \mathrm{ml}$ for P. earuginosa). 
Table 3. Combined effects of Ciprofloxacin and MF against Enterococcus faecalis as determined by Checkerboard method.

\begin{tabular}{l|cccccc}
\hline \multicolumn{1}{l}{$\begin{array}{l}\text { Combination } \\
\text { ratio }\end{array}$} & \multicolumn{2}{c}{ MF } & & ME \\
\hline & P. aeruginosa & P. mirabilis & E. faecalis & P. eruginosa & P. mirabilis & E. faecalis \\
\cline { 2 - 7 } & FIC Index & FIC Index & FIC Index & FIC Index & FIC Index & FIC Index \\
\hline $10: 0$ & - & - & - & - & - & - \\
$9: 1$ & $1.85^{\mathrm{c}}$ & $4.29^{\mathrm{d}}$ & $0.94^{\mathrm{a}}$ & $0.88^{\mathrm{a}}$ & $2.01^{\mathrm{d}}$ & $0.98^{\mathrm{a}}$ \\
$8: 2$ & $3.46^{\mathrm{d}}$ & $7.69^{\mathrm{d}}$ & $0.46^{\mathrm{a}}$ & $0.84^{\mathrm{a}}$ & $15.20^{\mathrm{d}}$ & $3.98^{\mathrm{d}}$ \\
$7: 3$ & $6.20^{\mathrm{d}}$ & $6.69^{\mathrm{d}}$ & $3.39^{\mathrm{d}}$ & $3.10^{\mathrm{d}}$ & $13.55^{\mathrm{d}}$ & $1.99^{\mathrm{c}}$ \\
$6: 4$ & $5.60^{\mathrm{d}}$ & $6.55^{\mathrm{d}}$ & $1.59^{\mathrm{c}}$ & $0.62^{\mathrm{a}}$ & $5.95^{\mathrm{d}}$ & 15.97 \\
$5: 5$ & $10.00^{\mathrm{d}}$ & $5.00^{\mathrm{d}}$ & $1.50^{\mathrm{c}}$ & $2.59^{\mathrm{d}}$ & $2.37^{\mathrm{d}}$ & $2.00^{\mathrm{c}}$ \\
$4: 6$ & $6.80^{\mathrm{d}}$ & $8.70^{\mathrm{d}}$ & $1.39^{\mathrm{c}}$ & $1.10^{\mathrm{c}}$ & $4.05^{\mathrm{d}}$ & $7.98^{\mathrm{d}}$ \\
$3: 7$ & $3.80^{\mathrm{d}}$ & $7.15^{\mathrm{d}}$ & $5.20^{\mathrm{d}}$ & $0.95^{\mathrm{a}}$ & $6.45^{\mathrm{d}}$ & $0.50^{\mathrm{a}}$ \\
$2: 8$ & $3.20^{\mathrm{d}}$ & $5.35^{\mathrm{d}}$ & $2.39^{\mathrm{d}}$ & $0.40^{\mathrm{a}}$ & $4.55^{\mathrm{d}}$ & $2.01^{\mathrm{d}}$ \\
$1: 9$ & $2.60^{\mathrm{d}}$ & $5.56^{\mathrm{d}}$ & $2.21^{\mathrm{d}}$ & $0.65^{\mathrm{a}}$ & $1.40^{\mathrm{c}}$ & $1.00^{\mathrm{b}}$ \\
\hline
\end{tabular}

$\mathrm{a}=$ Synergism $(\mathrm{F}<1) ; \mathrm{b}=$ Additivity $(\mathrm{F}=1) ; \mathrm{c}=$ Indifference $(\mathrm{F}>1 \leq 2)$;

$\mathrm{d}=$ Antagonism $(\mathrm{F}>2) ;{ }^{*}$ combination of Ciprofloxacin:MF or Ciprofloxacin:ME

Table 4. Antimicrobial activity of Ciprofloxacin disc and methanol crude extract (ME) of Phyllanthus muellerianus against the test organisms using the overlay inoculum susceptibility disc method.

\begin{tabular}{lcccc}
\hline $\begin{array}{l}\text { Test } \\
\text { organism }^{\text {a }}\end{array}$ & Test plate & $\begin{array}{c}\text { Cipro } \\
\text { (control plate) }\end{array}$ & \% Increase & Effect \\
\hline P. aeruginosa 1 & - & - & - & - \\
2 & - & - & - & - \\
3 & 9.00 & 20.00 & - & Antagonism \\
4 & 26.00 & 23.00 & 13.04 & Additivity \\
5 & - & 18.00 & - & - \\
P. mirabilis 1 & 20.00 & 15.00 & 33.33 & Synergism \\
2 & 20.00 & 37.00 & - & Antagonism \\
3 & 15.00 & 41.00 & - & Antagonism \\
4 & 18.00 & 27.00 & - & Antagonism \\
5 & 22.00 & 25.00 & - & Antagonism \\
E. faecalis 1 & 14.00 & 15.00 & - & Antagonism \\
2 & 15.00 & 13.00 & 15.38 & Additivity \\
3 & - & - & - & - \\
4 & 20.00 & 31.00 & - & Antagonism \\
5 & - & 12.00 & - & Antagonism \\
\hline
\end{tabular}

${ }^{a}$ The various isolates of each microorganism are numbered $1,2 \ldots 5$.

\section{MIC determination}

The MIC values of the plant extract (ME) and fraction (MF) that showed antimicrobial activities together with the standard reference antibiotic against the test organisms are presented in Table 2. These values ranged from $12.41 \pm 3.49$ (P. mirabilis) to $21.05 \pm 4.93 \mathrm{mg} / \mathrm{ml}$ (P. aeruginosa) and $11.41 \pm 1.99$ ( $P$. aeruginosa) to $17.53 \pm 1.43 \mathrm{mg} / \mathrm{ml}$ (P. mirabilis) for MF and ME respectively while the MIC of ciprofloxacin ranged from $0.03 \pm 0.01$ (P. mirabilis) to $0.33 \pm 0.05 \mathrm{mg} / \mathrm{ml}$ ( $E$. faecalis). The MIC values against the three microorganisms were significantly $(p<0.05)$ different from the MIC produced by ciprofloxacin against the organisms.

Interaction study of the test agents and ciprofloxacin The results of the in vitro evaluation of MF or ME and ciprofloxacin against the test organisms as determined by Checkerboard method are presented in Table 3. All the combination ratios with MF produced antagonism against P.aeruginosa. Indifference and antagonism were also produced by most of the combination ratios (Cipro: MF) against $P$. mirabilis and $E$. faecalis but synergy was observed at the ratios of 9:1 and 8:2 against $E$. faecalis. Most of the combination ratios of ciprofloxacin with ME (Cipro:ME) produced synergy against $P$. aeruginosa while the ratios of 9:1 and 3:7 produced synergy against E. faecalis. No synergy was observed in all the ratios (Cipro:ME) against P.mirabilis. The antimicrobial activity of ciprofloxacin disc (0.05) and ME against five isolates of each test microorganism using the Overlay Inoculum susceptibility disc method shows that the combination produced antagonism against most isolates of the test organisms (Table 4). The combination of ciprofloxacin and ME did not produce synergistic effect against any of the isolates of $P$. aeruginosa or E. faecalis. However, additivity was observed against some of the isolates of the two organisms while synergy was produced only against one of the isolates of $P$. mirabilis.

\section{Discussion}

Antimicrobial substances are desirable tools in the control of undesirable microorganisms especially in the treatment of infections and in preservation of food. The active components usually interfere with the growth or metabolism of microorganisms in a negative manner. The crude extract, $M E$, 
produced higher antibacterial activity against $P$. aeruginosa, P. mirabilis and E. faecalis than MF as shown by the IZD and MIC values. The effect produced by ME is however lower than that of the standard drug but this suggests that higher concentrations of the crude ME could produce comparable antimicrobial results. The antimicrobial activity of an agent is usually quantified by determining the MIC values which serve as a guide for treatment of most infections. Though the three microorganisms proved sensitive to the plant extracts, the most sensitive organism was $P$. aeruginosa. Fractionation or purification of the methanol extract using methanol reduced the antibacterial effect of the leaf of plant as shown in Table 1. It is possible that the antibacterial principles reside within the secondary metabolites and the effects are more when used together than when used singly. $P$. aeruginosa is an opportunistic, nocosomial pathogen of immuno-compromised individuals. The organism typically infects the pulmonary tract, urinary tract, burns, wounds, and also causes other blood infections. It is the most common cause of infections of burn injuries and of the outer ear (otitis externa), and is the most frequent colonizer of medical devices (e.g., catheters) E. faecalis can cause endocarditis, as well as bladder, prostate, and epididymal infections [22]. Thus the methanolic extract of the plant can be used to treat infections caused by P.aeruginosa, $P$.mirabilis and E. faecalis organisms. In addition, the fact that none of the fractions or extract produced antibacterial effect on Klebsiella spp., E.coli, Salmonella spp. and Candida albicans suggests that the leaf of $P$. muellerianus may not be effective against infections caused by these organisms. It is probable the plant leaf extract does not possess does not possess any activity against fungi.

The antimicrobial activities of the plant extract and fraction can be attributed to the presence of secondary metabolites including glycosides, alkaloids, steroids, terpenoids, flavonoids and saponins. The microorganisms were sensitive to ME and MF which were rich in flavonoids that have the ability to complex with extracellular and soluble proteins and with microbial cell wall $[\mathbf{2 3}, \mathbf{2 4}]$. Glycosides are plant constituents used as purgatives for either habitual constipation or occasional use and in the treatment of diarrhea. Alkaloids have been demonstrated to possess many biological activities which include analgesic, antiplasmodic, bactericidal [25] and antifungal effect and they also have the ability to intercalate between DNA strands. Saponins are also known to intercalate DNA of microorganisms and this is the basis of their antimicrobial activity [26]. These constituents acting alone or in combination could be responsible for the observed effect of this plant.

The result of the interaction study of the plant extract or fraction with ciprofloxacin showed indifference and antagonism in most cases against $P$. mirabilis and $E$. faecalis. These suggest that the combination of the plant extract (ME or MF) with ciprofloxacin could reduce or may not affect the potency of either agent against the organisms. Thus, the combination of the plant extract with ciprofloxacin may not possess any advantage over any agent used alone against P.mirabilis and E. faecalis. Decreased activity may be attributed to phytochemical incompatibility between the combined agents or a competitive inhibition at the site of action. Synergy was observed in most of the combination ratios with $\mathrm{ME}$ against $P$. aeruginosa indicating that the organism is the most sensitive to the leaf extract of P. muellerianus. The results suggest that it could be more therapeutically beneficial to use the combined extract and ciprofloxacin against infections caused by $P$. aeruginosa. In that case, greater antibacterial effect could be obtained at lower doses of each agent thereby minimizing their possible adverse effects. The results shown by the overlay inoculum susceptibility agar technique is similar to that shown by the Checkerboard technique with antagonism being the predominant effect. Synergism and additivity were, however, observed in some cases. The results are further indication that combination of the plant extract with ciprofloxacin at certain ratios could be better than any of the agents used alone.

\section{Conclusion}

The present investigation indicates that the methanol crude extract and methanol fraction of the leaves of Phyllanthus muellerianus have promising antimicrobial activities against the $P$. aeruginosa, $P$. mirabilis and $E$. faecalis but no activity against Klebsiella spp., E.coli, Salmonella spp. and Candida albicans. Combinations of the methanol extract or fraction of the leaf of the plant with ciprofloxacin showed no added advantage over each agent used alone against $P$. mirabilis and $E$. faecalis while certain combination ratios are better against $P$. aeruginosa. The antibacterial activity of the leaf extract is possibly due to the presence of some secondary metabolites such as flavonoids, saponins and glycosides. Using the crude methanol extract (ME) of the leaf appeared to produce better antimicrobial effect than the purer form (MF). Further purification of the extract is however necessary in order to isolate and characterize the active principles.

\section{Competing interests}

The authors declare that they have no competing interests.

\section{Authors' contributions}

OKC conceived and participated in the design of the study, analysis and interpretation of the data and in drafting the manuscript. AAA identified the plant species and participated in the design of the study, analysis and interpretation of the data and in drafting the manuscript. UPF participated in the analysis and interpretation of the data and in drafting the manuscript. OMO participated in carrying out the study and in analysis and interpretation of the data. All authors read and approved the final manuscript.

\section{Publication history}

Received: 11-Dec-2012 Revised: 12-Feb-2013

Re-Revised: 08-Mar-2013 Accepted: 02-Apr-2013

Published: 16-Apr-2013 
Ofokansi et al. Journal of Pharmaceutical Technology \& Drug Research 2013, http://www.hoajonline.com/journals/pdf/2050-120X-2-16.pdf

\section{References}

1. Iwu MM, Duncan AR, Okunji CO: New antimicrobials of plant origin: Perspectives on new crops and new uses. Alexandra, ASHS Press 1999. I Pdf

2. Kone WM, Atindehou KK, Kacou-N'douba A and Dosso M: Evaluation of 17 medicinal plants from Northern Cote d'Ivoire for their in vitro activity against Streptococcus pneumoniae. Afr J Tradit Complement Altern Med 2006, 4:17-22. | Article | PubMed Abstract I PubMed Full Text

3. Pinner RW, Teutsch SM, Simonsen L, Klug LA, Graber JM, Clarke MJ and Berkelman RL: Trends in infectious diseases mortality in the United States. JAMA 1996, 275:189-93. | Article I PubMed

4. Cos PM, Sindambiwe LW, Vlietink AJ, Berghe DV: Bioassays for antimicrobial and antifungal activities. In: Biological screening of plant constituents. Edited by Mahabir P, Gupta S, Swami H, Karan V. Trieste, International Centre for Science and High Technology 2006, 19-28.

5. Thomson WAR: Medicines from the Earth propilis and antimicrobial drugs. Microbiol Res 1978, 158: 353-357.

6. Assob JC, Kamga HL, Nsagha DS, Njunda AL, Nde PF, Asongalem EA, Njouendou AJ, Sandjon B and Penlap VB: Antimicrobial and toxicological activities of five medicinal plant species from Cameroon traditional medicine. BMC Complement Altern Med 2011, 11:70. | Article | PubMed Abstract | PubMed Full Text

7. Ofokansi KC, Eze AO, Uzor PF: Evaluation of the antimicrobial activity of the aqueous and methanolic leaf extracts of Mitacarpus villosus with amoxicillin. African J Pharm Res Dev 2011, 3(1): 43-47.

8. Diallo D, Hveen B, Mahmoud MA, Betge G, Paulson BS, et al: An ethnobotanical survey of herbal drugs of Gourma district, Mali. Pharm Biol 1999, 37:80-91. I Article

9. Burkill HM: The Useful Plants of West Tropical Africa. 2nd ed., Vol.2, United Kingdom, Families E-1 Royal Botanic Gardens, 1995.

10. Arbonnier M: Trees, Shrubs and liana of West African dry zones. Paris, France, Margraf Publishers 2004.

11. Doughari JH, Sunday D: Antibacterial Activity of Phyllanthus muellerianus. Pharm Biol 2008, 46 (6): 400-405. | Article

12. Katsayal UA, Lamal RS: Preliminary phytochemical and antibacterial screening of the ethanolic stem bark extract of Phyllanthus muellerianus. Nigeria J Pharm Sci 2009, 8(2): 121-125.

13. Sofowora A: Medicinal Plants and Traditional Medicine in Africa. 3rd ed., Nigeria, Spectrum Books Ltd. 2008.

14. Evans WC: Trease and Evans Pharmacognosy. 15th ed., London, W. B, Saunders Company Ltd. 2002.

15. Cheesbrough M: District Laboratory Practice in Tropical Countries Part 2. Cape Town 8001, S/Africa, Cambridge University Press 2000.

16. Cowan SI, Steel KJ: Cowan and Steel's Manual for the Identification of Medical Bacteria. Barrow, U.S.A 1993. I Book

17. Arora DR: Textbook of Microbiology. 2nd ed., New Delhi, CBS Publishing Company 1999. I Book

18. Mirjana MS, Marija M, Dusica M: Antibacterial and antifungal activities of Isatin N mannich bases. J. Pharm. Sci 1979, 68(4): 45962.

19. Rios JL, Recio MC and Villar A: Screening methods for natural products with antimicrobial activity: a review of the literature. $J$ Ethnopharmacol 1988, 23:127-49. | Article | PubMed

20. National Committee for Clinical Laboratory Standards (NCCLS): Performance standards for antimicrobial susceptibility testing. 9th ed., Wayne 1999.

21. Okore VC: Principles of Pharmaceutical Microbiology. 2nd ed., Nigeria, Ephrata Publishers 2009.

22. Anitha VT, Antonisamy JM and Jeeva S: Anti-bacterial studies on Hemigraphis colorata (Blume) H.G. Hallier and Elephantopus scaber L. Asian Pac J Trop Med 2012, 5:52-7. I Article I PubMed

23. Houghon $P$, Hawkes PR, Jane E: Naphtoquinones and an alkaloid from root of Newboulbia laeves. Phytochem 1994, 35:1602-1603.

24. Tsuchiya H, Sato M, Miyazaki T, Fujiwara S, Tanigaki S, Ohyama
M, Tanaka T and linuma M: Comparative study on the antibacterial activity of phytochemical flavanones against methicillin-resistant Staphylococcus aureus. J Ethnopharmacol 1996, 50:27-34. | Article | PubMed

25. Okwu DE: Phytochemicals and vitamin contents of indigenous specie of South East Nigeria. J Sustain Agric Environs 2004, 6(2): 140-147.

26. Phillipson JD, O'Neill MJ: New leads to the treatment of protozoal infections based on natural product molecules. Acta Pharm Nord 1987, 1:131-144.

Citation:

Ofokansi K C, Attama A A, Uzor P F and Ovri M O: Evaluation of the combined antimicrobial activity of the leaf extract of phyllantus muellerianus with ciprofloxacin. journal of Pharmaceutical Technology and Drug Research 2013, 2:16. http://dx.doi.org/10.7243/2050-120X-2-16 